\title{
Use of Focused Ion Beam as a Sample Preparation Tool for Cryo-Electron Tomography
}

\author{
Samuel Záchej ${ }^{1}$, Jana Havránková ${ }^{2}, K_{\text {ristýna Rosíková }}$, Rostislav Váňa ${ }^{1}$, and Miloslav Havelka ${ }^{1}$ \\ 1. TESCAN Brno, Brno, Czech Republic \\ 2. TESCAN ORSAY HOLDING, Brno, Czech Republic
}

Cryo-electron tomography is increasingly being used as a powerful tool to study molecules, macromolecular complexes and the ultrastructure of various cells and tissues. Cryo-ET is based on the imaging techniques and image processing methods of a TEM, mainly Single Particle Analysis or 3D reconstruction from a tilt series. The strength of the method depends largely on the quality of the specimen, preservation of a near-native state and crucially, the thickness, which is critical for quality reconstruction. For optimal results, the sample must be thinner than $500 \mathrm{~nm}$ [1]. However, most biological samples such as bacteria or eukaryotic cells and tissues are much thicker. In terms of sample preparation techniques, cryo-sectioning or cryo-ultramicrotomy is a well-known method used for thinning such samples. However, the technique suffers from multiple artefacts, which can adversely affect data quality. The most serious defects are caused by compression, knife-marks and curtains or problems with fixing and placing the slices on the TEM sample holder [2].

Over the last few years a focused ion beam (FIB) has been employed for sample preparation under cryogenic conditions. Thinning of a vitrified biological specimen by means of a FIB is artefact-free and can thus substitute cryo-sectioning. When done precisely, cryo-FIB thinning can deliver a thin, vitrified sample lamella in a close-to-native state without any mechanical distortions that is ideal for TEM imaging and tomography.

Here we present the entire workflow of site-specific FIB sample preparation for cryo-ET. As a standard sample for optimization of the whole workflow, we used Saccharomyces cerevisiae applied to a standard TEM grid (300 mesh covered with carbon and formvar foil). Yeast cells were frozen by a simple plunging into liquid ethane for fast and deep vitrification of the hydrated sample. The frozen sample was attached to the special on-grid lamella sample holder and to enhance surface conductivity, the prepared sample was sputter-coated with a 10-nm layer of organometallic platinum. The actively cooled sample was transferred to the TESCAN S8000G equipped with the new ORAGE FIB which provides excellent beam properties at low currents. The accuracy of the low-current FIB machining is extremely important for quality of the final lamella. To maintain the sample at cryogenic temperatures during milling, the instrument is equipped with a cryo-stage and a cryo-shield.

Sample processing inside the SEM chamber began by depositing a protection layer above the region of interest (ROI). A platinum-containing precursor is condensed on the sample surface with the Gas Injection System and scanned using a low-current ion beam $(150 \mathrm{pA})$, until the sponge-like condensate forms a firm protective layer [3]. This protection layer serves as a shield during the milling and polishing phases and thereby eliminates curtaining of the milled surface due to irregularities in the sample. Then the ORAGE FIB is used to mill a trench from the top and bottom of the selected plane to specifically uncover the internal structure of the cells. The maximum milling current must be kept low (usually lower than $500 \mathrm{pA}$ ), to avoid damaging the sample (mainly recrystallisation). The lamella is then polished and thinned down to $300 \mathrm{~nm}$ using low-current ion beam (10 pA). Figure 1 shows the 
resulting polished lamella. The milling and polishing process is controlled by a vector-based scanning generator DrawBeam ${ }^{\mathrm{TM}}$ which allows standardization of the whole workflow into a single semiautomated project. Once the lamella is polished, the sample holder with the sample can be transferred directly to the cryo-TEM holder for further inspection (e.g. cryo-ET or single particle analysis). The presented workflow can be used as a routine method to prepare samples for cryo-TEM applications.

\section{References:}

[1] Zhang Jianguo et al, Journal of Structural Biology, Vol. 194 (2016), p. 218-223

[2] A.Al-Amoudi, D. Studer and J. Dubochet, Journal of Structural Biology, Vol. 150 (2005), p. 109-121

[3] M.F.Hayles et al, Journal of Microscopy, Vol. 226 (2007), 263-269

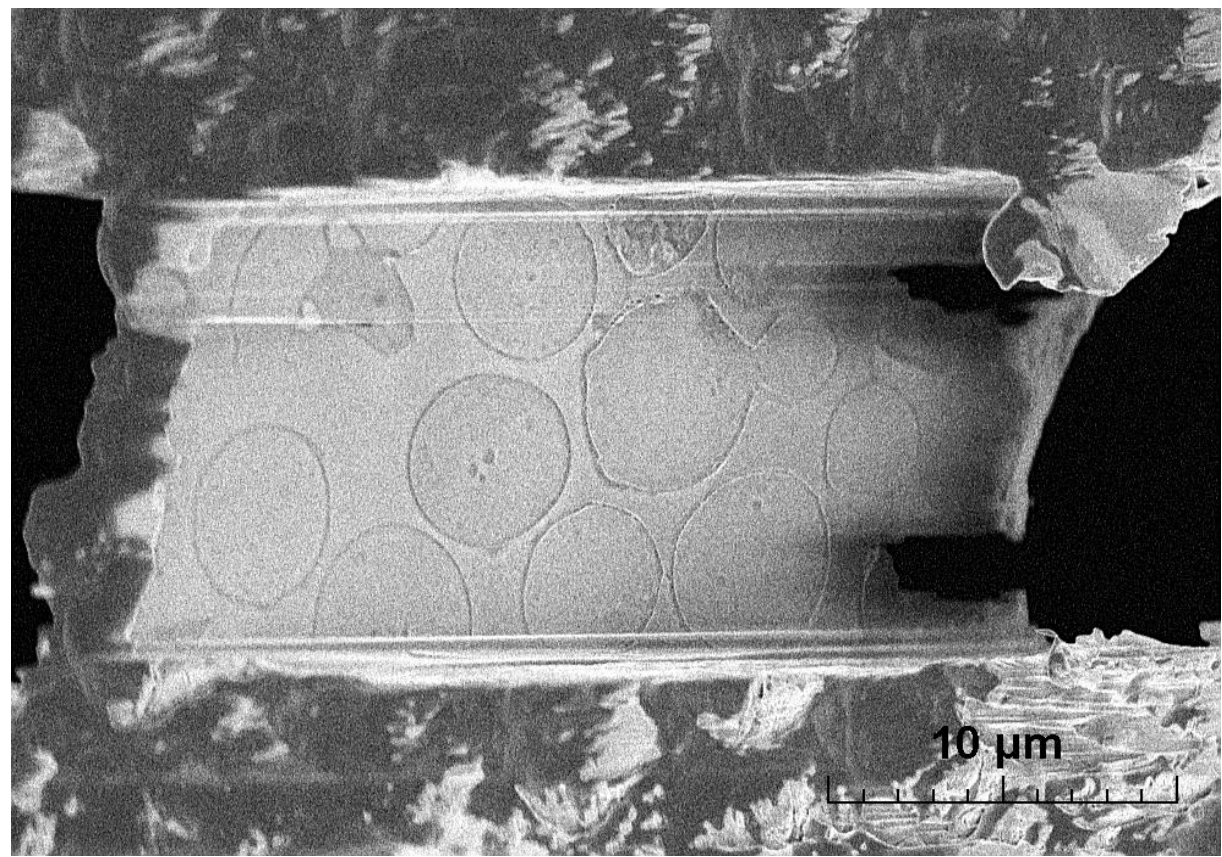

Figure 1. Top view of the $300 \mathrm{~nm}$ thick lamella prepared from a yeast culture. The top and bottom surface of the lamella is almost perfectly flat. 